\title{
TIC en padres para mejorar el rendimiento académico
}

\author{
Mg. Jessica Guerra Pérez \\ salvadorayurimaguas93@gmail.com \\ Escuela de posgrado \\ Universidad César Vallejo \\ ORCID: 0000-0003-4931-3844 \\ Dr. José Manuel Delgado Bardales \\ jmdelgadob@ucvvirtual.edu.pe \\ Escuela de posgrado \\ Universidad César Vallejo \\ ORCID:0000-0001-6574-2759 \\ Scopus autor ID: 24070333700 \\ Código Renacyt: P0050554
}

\section{RESUMEN}

La investigación tuvo como objetivos, Caracterizar las tecnologías de información y comunicación en padres para en el rendimiento académico de los estudiantes La investigación fue básica de diseño descriptivo con revisión sistemática, las revisiones sistemáticas son investigaciones científicas en las cuales la unidad de análisis son los estudios originales primarios, constituyen una herramienta esencial para sintetizar la información científica disponible. Se encontró que las Tecnologías de Información y Comunicación se encuentran integradas en las familias a nivel internacional, nacional, local urbana y rural, especialmente a través del celular. La actuación de los padres con sus competencias parentales contribuye al desarrollo sostenido de los niños y niñas a su cargo, que se prolonga a través de toda la vida en especial la vida escolar. Integrar las TIC a los padres para ejercer competencia parental en la escuela y ésta evidenciarse en el buen rendimiento académico de los estudiantes es un aspecto necesario. Actualmente se reconoce el papel de las TIC y el apoyo de los padres como elementos fundamentales para llevar adelante la educación remota por tiempos de pandemia; y, aún más convertirse en un aliado para apoyar en la educación de los hijos, evidenciado en el mejor rendimiento académico.

Palabras clave: Tecnología, información y comunicación, rendimiento académico 


\title{
ICT in parents to improve academic performance, 2020
}

\begin{abstract}
The research aimed at characterizing information and communication technologies in parents for the academic performance of students The research was basic of descriptive design with systematic review, systematic reviews are scientific investigations in which the unit of analysis are studies Primary originals are an essential tool to synthesize the available scientific information. It was found that Information and Communication Technologies are integrated into international, national, local urban and rural families, especially through cell phones. The performance of parents with their parental skills contributes to the sustained development of the children in their charge, which lasts throughout life, especially school life. Integrating ICT with parents to exercise parental competence at school and this evidenced in the good academic performance of students is a necessary aspect. Currently, the role of ICTs and the support of parents are recognized as fundamental elements to carry out remote education in times of pandemic; and, even more, to become an ally to support the education of their children, evidenced in the best academic performance.
\end{abstract}

Keywords: Technology, information and communication, academic performance

Artículo recibido: 03 nov. 2020 Aceptado para publicación: 07 dic. 2020 Correspondencia salvadorayurimaguas93@gmail.com Conflictos de Interés: Ninguna que declarar 


\section{INTRODUCCIÓN}

La educación es importante para el progreso de un país porque contribuye al desarrollo de los ciudadanos, sin embargo, demanda trabajo conjunto de los actores que intervienen directamente en éste proceso, es decir, estudiantes, docentes, padres de familia. En Perú, hasta ahora se ha priorizado la creación de estrategias que facilitan la acomodación significativa de aprendizajes en los estudiantes y la mejora de las metodologías de enseñanza en los docentes, pero continuamos con serias deficiencias, según lo muestra los resultados en las evaluaciones internacionales del Programme for International Student Assessment - PISA(2016), que nos coloca en el puesto 64 en el área de ciencia, puesto 63 de comprensión lectora y puesto 64 en el área de matemática de 70 países participantes, pese a ello tenemos el mayor crecimiento histórico.

Por otra parte las tecnologías de información y comunicación son parte de la vida cotidiana, desde su expansión en la década de los 80 y el enfoque nanotecnológico que abarataron los costos, haciéndolos asequibles, muchos padres las integran, según la Unión Internacional de Telecomunicaciones - UIT (2016), organismo especializado de las Naciones Unidas para las tecnologías de la información y la comunicación-TIC reporta que en el mundo, casi 1.000 millones de personas tienen acceso a Internet (de los cuales 230 millones están en China, 60 millones en la India y 20 millones en los 48 Países Menos Adelantados del mundo), eliminando barreras temporales y territoriales, dando pase a la tan laureada globalización.

Los padres de familia no son ajenos al cambio que se exige, pues al no hacerlo corren el riesgo de desaparecer virtualmente, enfrentarse a ser analfabetos digitales y no cumplir a cabalidad los roles escolares de supervisión que deben realizar, especialmente en el ámbito escolar, la verdadera integración de los padres en la escuela es muy importante, ya que contribuye al noble propósito de la educación, pero los padres no interiorizan en su totalidad, ese pensamiento y se observa en las escuelas especialmente secundarios de la selva del Perú, un gran ausentismo que se agudiza en los años pertenecientes al VII Ciclo, es decir, tercero, cuarto, quinto, donde los jóvenes, cuyas edades oscilan entre los 14,15 y 16 años de edad responden personalmente por los estudios que realizan, la participación de los padres es poca o nula.

La temática presentada ha sido ampliamente estudiada, respecto a las TIC, tal es el caso de Colas P. y Contreras J. (2013), Lozano J., Ballesta J., Alcaraz S. y Cerezo C. (2014), 
Para Hernández A., López P., Sánchez S. (2014), Macià M. (2016), los autores consideran que las TIC son elemento importante y cada vez forma parte integrante de la familia y del quehacer cotidiano.

Llevot N. y Bernad O. (2015), Garreta J. (2016), Pedraza C., Amado O. y Munévar P. (2019), por su parte mencionan que la integración del padre de familia en la educación de sus hijos es fundamental da la posibilidad de ejercer un control parental implícito y explícito y ello se refleja en el rendimiento académico, las posibilidades que las TIC ofrecen para éste propósito son infinitas por tanto está en el actuar de los padres la eficiencia y eficacia de éstas herramientas, sin embargo, el panorama que encontramos especialmente en las escuelas rurales de Perú son de rechazo, naturalmente por la poca o nula habilidad que se tiene en el más elemental de los dispositivos como lo es el celular. Por su parte Ramírez A. (2020), en el estudio realizado enfatiza que, las familias muestran interés moderado por las TIC en especial para el aprendizaje de estrategias de seguimiento del uso de los dispositivos móviles con menores, con el claro objetivo de brindar las facilidades a sus hijos. Con la situación de pandemia que ahora vivimos y al haber establecido en Perú, como en muchas partes del mundo, la educación remota, mediante TIC, las carencias son notorias, realidad concordante con otros países, según lo señala Mirta Britez (2020), cuando concluye se ha sacado a luz aspectos importantes a mejorar para impartir clases a distancia haciendo uso de los entornos virtuales de aprendizaje como son: los padres sin preparación para acompañar en las tareas a sus hijos, el desconocimiento en el uso de los ambientes virtuales, padres que no saben usar la plataforma. Estudio fue realizado en los países vecinos de Paraguay, Brasil y Argentina. Es importante mencionar el estudio realizado por García A. y Barreto M. (2014) que concluyó tanto en zona rural como en urbana en Perú, que los hombres y las mujeres en edad escolar usan las TIC mayormente como entretenimiento recubierto con la necesidad de usarlo para la escuela, con ese pretexto sus padres les dan permiso y el dinero para acceder a las cabinas públicas, situación que es ignorada por los padres, quienes en cumplimiento de sus funciones paternales y anhelos de progreso para sus hijos les brindan dinero y permiso para acceder a las cabinas públicas.

\section{ESTRATEGIAS METODOLÓGICAS O MATERIALES Y MÉTODOS}

El tipo de investigación es básica de diseño descriptivo con revisión sistemática, las revisiones sistemáticas son investigaciones científicas en las cuales la unidad de análisis 
son los estudios originales primarios, constituyen una herramienta esencial para sintetizar la información científica disponible incrementa la validez de las conclusiones de estudios individuales e identificar áreas de incertidumbre donde sea necesario realizar investigación Hernández (2016, p.217). Es descriptiva porque se recogió información relacionada a las Tecnologías de la Información y la Comunicación con padres para mejorar el rendimiento académico de los estudiantes. Por lo mencionado se revisó información científica y se visualizó como solucionar una necesidad que radica en la integración de los padres a las escuelas eliminando barreras temporales y geográficas mediante el uso de las TIC - específicamente los celulares inteligentes.

\section{RESULTADOS Y DISCUSIÓN}

La recolección de información se realizó a través de una tabla de registro de datos y la fuente fue revisión bibliográfica de artículos de investigaciones tanto nacionales como internacionales que tuvieron como tema principal a las Tecnologías de la Información y la Comunicación con padres para mejorar el rendimiento académico de los estudiantes; de todos los artículos que se encontraron se incluyeron los estudios (10) más importantes según nivel de evidencia y se excluyeron los menos relevantes. Se estableció la búsqueda siempre y cuando las características en las cuales concuerda y los puntos en los que existe discrepancia entre artículos nacionales e internacionales, además de acuerdo a criterios técnicos preestablecidos, se realizó una evaluación crítica e intensiva de cada artículo, a partir de ello, se determinó la calidad de la evidencia y la fuerza de recomendación para cada artículo.

Posterior a la revisión sistemática de los artículos del 100\%, el 90\% corresponde a estudios internacionalmente realizados en España y países latinoamericanos, el 10\% realizado en el Perú. En relación a los diseños y tipos de estudios el 90\% son descriptivos y $10 \%$ es sistemático y descriptivo.

En relación a las variables, TIC en padres, el autor Colás P. Contreras J (2013), en el estudio denominado "La participación de las familias en los centros de educación primaria", cuyo Tipo y diseño de la investigación fue Descriptivo cuantitativo, la población y muestra 110 familias de alumnos de primaria que representa a una población 41.799 familias sevillanas, el instrumento utilizado fue el cuestionario ad hoc basado en la conjunción de los modelos teóricos de Vogels y Epstein, el estudio se realizó teniendo en cuenta el Código de ética en investigación. Los resultados muestran las preferencias 
por unas determinadas opciones de colaboración, así como la coexistencia de concepciones y motivaciones que representan distintos niveles de implicación y compromiso con la educación. Para finalmente concluir que la participación de las familias en la escuela es un indicador relevante de la calidad del Sistema Educativo. Sin embargo, la participación de las familias en los centros educativos es baja en determinadas ofertas de participación, y positiva y/o activa en otras.

Por su parte Lozano J., Ballesta J, Alcazar S., Cerezo C. (2014), en el trabajo de investigación llamado "Las tecnologías de la información y la comunicación en la relación familia - escuela", cuyo Tipo y diseño de la investigación es transversal, la población y muestra 1932 familias de la Región Autónoma de Murcia, el instrumento utilizado fue el cuestionario, el estudio se realizó teniendo en cuenta el Código de ética en investigación. En los resultados los autores mencionan que "...se constata una necesidad de profundizar en torno a esta temática, para llegar a comprender y valorar el papel que tiene la familia en el uso de las TIC y su importancia dentro del ámbito escolar, ya que las familias pueden condicionar el uso de estos medios a favor de los alumnos. Sin olvidar, que hoy nos encontramos ante una sociedad digital donde toda la comunidad educativa, a nivel de usuario, necesita comunicarse, a través de estas tecnologías. Este hecho supondrá que es necesario trabajar de forma coordinada familia-escuela, puesto que la actitud de ambos colectivos va a influir en el desarrollo personal y social de nuestros alumnos. Para finalmente concluir que la participación de las familias en la escuela es un indicador relevante de la calidad del Sistema Educativo. Sin embargo, la participación de las familias en los centros educativos es baja en determinadas ofertas de participación, y positiva y/o activa en otras. Los autores remarcan que es necesario profundizar en el estudio de ésta temática, claramente se reconoce el potencial de las TIC para el trabajo de supervisión de los padres en beneficio de los estudiantes.

Hernández A., López P., Sánchez S. (2014), en el estudio "La comunicación en la familia a través de las TIC. Percepción de los adolescentes", cuyo Tipo y diseño de la investigación es descriptivo, la población y muestra 01 centro de secundaria, el instrumento utilizado fue el cuestionario, el estudio se realizó teniendo en cuenta el Código de ética en investigación. En los resultados describen el escenario conocido “...una alta integración de las TIC en los hogares, un escaso uso de las mismas para comunicarse con sus progenitores, y una relación paterno-filial hacia las TIC 
caracterizada por el establecimiento de normas y la permisividad hacia las mismas”. El estudio concluye que las familias se integran a las TIC, los padres se limitan a establecer normas y permisos a sus hijos para el uso de ellas, sin embargo, es necesario se cumpla a cabalidad el control parental para asegurar el buen rendimiento académico de sus hijos. Para García A., Barreto M. (2014), en el trabajo de investigación "El uso, apropiación e impacto de las TIC por las mujeres rurales jóvenes en el Perú”, cuyo Tipo y diseño de la investigación es descriptivo - cualitativo, la muestra 02 centros poblados rurales de Perú denominado Nuevo Pedregal y Andaray, el instrumento utilizado fue entrevista a profundidad, el estudio se realizó teniendo en cuenta el Código de ética en investigación. En los resultados Respecto a lo pertinente con el tema que se investiga el resultado relevante lo menciona diciendo que “...en los hombres, las mujeres usan estas herramientas como entretenimiento, aunque recubierto con un discurso que pasa por la escuela y los deberes escolares, que permiten afirmar la "necesidad" de usar de Internet. Es con ese pretexto que sus padres les dan permiso y el dinero para acceder a las cabinas públicas (sin que ello signifique que no sea verdad que hagan tareas utilizando la web). En nuestro país según lo investigado por las autoras, el uso de las TIC (cristalizado en el celular) se utiliza mayormente para entretenimiento, ello es ignorado por los padres, quienes en cumplimiento de sus funciones paternales y anhelos de progreso para sus hijos les brindan dinero y permiso para acceder a las cabinas públicas, lo que da a conocer nuevamente la importancia del presente estudio a fin de integrar las TIC a los padres en beneficio de los estudiantes visualizado en su rendimiento académico.

Llevot N., Bernard O. (2016), el estudio llamado "La participación de las familias en la escuela : Factores clave", cuyo Tipo y diseño de la investigación es descriptivo, la población y muestra 353 escuelas públicas, 22 docentes, 17 progenitores distribuidas por todo el territorio de Cataluña, el instrumento utilizado fue encuesta telefónica, entrevista en profundidad y estudio etnográfico, el estudio se realizó teniendo en cuenta el Código de ética en investigación. En los resultados Lo evidenciado por las autoras es que existe integración de las TIC, sin embargo, su uso es mejorable en algunos centros, tanto por parte de los docentes como de las familias. La conclusión relevante con el estudio es : toda vez que los docentes son profesionales y pese a ello necesitan mejorar la interacción con las TIC, la situación se agudiza en el panorama de las familias, ya que muchas veces 
son de escasos recursos económicos que les impide adquirir el recurso TIC y por ende adquirir habilidades y destrezas en el manejo de ellos.

Asimismo, Macià M. (2016), en el estudio llamado "La comunicación familia escuela : el uso de las TIC en los centros de primaria", cuyo Tipo y diseño de la investigación es descriptivo - cualitativo, la población y muestra 20 centros educativos de 4 regiones : Cataluña, Aragón, La Rioja y las Islas Baleares, el instrumento utilizado fue entrevistas, fichas, el estudio se realizó teniendo en cuenta el Código de ética en investigación. El resultado dice que aunque existen docentes y progenitores que rechazan Taxativamente el uso de las plataformas y los mails para la comunicación entre escuela y familia, son muchos más los que tienen una actitud abierta hacia los mismos. La idea más importante es que maestros y docentes, tienen una actitud positiva y abierta hacia éstos. La autora considera que la idea más importante que se desprende de este estudio es que maestros y docentes, aunque a día de hoy no utilicen demasiado los canales de comunicación digitales, tienen una actitud positiva y abierta hacia éstos.

Garreta J. (2016), el estudio llamado "Fortalezas y debilidades de la participación de las familias en la escuela", cuyo Tipo y diseño de la investigación es descriptivo - cualitativo, la población y muestra 15 significativas entrevistas en España, el instrumento utilizado fue entrevistas a profundidad, el estudio se realizó teniendo en cuenta el Código de ética en investigación. El resultado indica que la participación de las familias en la escuela es considerada un factor de gran importancia en la educación del alumnado, sea por los beneficios en cuanto a éxito académico del alumnado, la conclusión indica que la participación de las familias en centros de infantil y primaria e institutos es importante, así como analizar las Fortalezas y debilidades de su situación actual.

Pedraza C., Amado O., Munévar P., (2019), el estudio titulado “Apropiación de dispositivos móviles en educación una experiencia de sistematización sobre uso pedagógico de TIC en tablets", cuyo Tipo y diseño de la investigación es descriptivo, la población y muestra Pauna, Municipio del Departamento de Boyacá, el instrumento utilizado fue análisis sistemático, los estudios realizados muestran el aporte de los autores para la presente investigación cuando indican •que se editó y socializó una cartilla digital de 5 unidades dónde La segunda unidad entre otros aspectos aborda aspectos relacionados con “... el control parental...”. Los autores de la investigación reconocen la importancia de integrar al padre las TIC, para ejercer de forma explícita o implícita el 
control parental para lograr el éxito de sus hijos evidenciado en el rendimiento académico que tienen.

Por su parte Ramírez A., Salcines I. Gonzáles N., (2020), el estudio titulado "Los dispositivos móviles en el hogar. Interés formativo de las familias españolas”, cuyo Tipo y diseño de la investigación es cuantitativo, la población y muestra 1082 integrantes de unidades familiares españolas, el instrumento utilizado fue cuestionario Ad hoc, los estudios muestran que las familias tienen un interés moderado en la formación sobre gestión de los dispositivos móviles con menores (55.7\%), la temática más aceptada por las familias es el aprendizaje de estrategias de seguimiento del uso de los dispositivos móviles", la conclusión relevante, la conclusión relevante es que se aprecia en la investigación que la forma de supervisar y acompañar a los estudiantes a través de las TIC es importante para las familias, lo que da relevancia al estudio que se viene realizando.

Britez M. (2020), el estudio titulado "La educación ante el avance del COVID-19 en Paraguay, comparativo con países de la triple Frontera", cuyo Tipo y diseño de la investigación es Descriptivo - comparativo, hermenéutico, la población y muestra Normativa de 03 países Paraguay, Brasil y Argentina, el instrumento utilizado fue Análisis de Documentos, los estudios muestran que la autora concluye que se han tomado las medidas necesarias para no perder el año escolar, pero esto ha sacado a luz aspectos importantes a mejorar para impartir clases a distancia haciendo uso de los entornos virtuales de aprendizaje. Importante el análisis realizado y es pertinente con el presente estudio especialmente cuando menciona que “...existen diferentes dificultades y mucho por mejorar en especial la actuación de los padres como lo señala la autora "Padres que no saben usar la plataforma" y “...Otro aspecto negativo, los padres sin preparación para acompañar en las tareas a sus hijos, el desconocimiento en el uso de los ambientes virtuales, la dificultad para usar el celular en el caso de varios niños, el costo de internet (caso subsanado en Paraguay).

Finalmente el Ministerio de Educación como ente rector de la educación Peruana ha emitido la Resolución Viceministerial $N^{\circ}$ 088-2020 Minedu que establece el trabajo remoto en las escuelas y la Resolución Viceministerial No 097-2020-Minedu que aprueba el documento Normativo para el trabajo remoto, con el fin de implementar el año lectivo 2020 sin poner en riesgo la salud de la comunidad educativa y donde el rol de los padres 
es importante porque actúa como principal aliado, especialmente en colaborar de manera directa en el proceso de enseñanza aprendizaje de sus hijos, constituyéndose un sólido apoyo en el trabajo.

Como se puede apreciar los distintos autores que hemos mencionado enfatizan la importancia de integrar las TIC en los actores educativos, para fines de la investigación, integrarlos especialmente a los padres de familia, con ello garantizar el adecuado uso, exploración de los recursos e información de fuentes confiables que permita lograr los fines de la educación. Al estar los estudiantes en crecimiento y fácilmente se distraen y concentran en el entretenimiento, en cierta medida recubierta de la necesidad escolar. Se hace necesario que los padres se familiaricen con las TIC y puedan realizar efectivamente el control parental.

\section{CONCLUSIÓN O CONSIDERACIONES FINALES}

1. Las Tecnologías de Información y Comunicación se encuentran integradas a las familias a nivel internacional, nacional, local urbanas y rurales, especialmente a través del celular.

2. La actuación de los padres con sus competencias parentales contribuye al desarrollo sostenido de los niños y niñas a su cargo, que se prolonga a través de toda la vida en especial la vida escolar.

3. Integrar las TIC a los padres para ejercer competencia parental en la escuela y ésta evidenciarse en el buen rendimiento académico de los estudiantes es un aspecto necesario de urgente atención.

4. Actualmente se reconoce el papel de las TIC y el apoyo de los padres como elementos fundamentales para llevar adelante la educación remota por tiempos de pandemia; y, aún más convertirse en un aliado para apoyar en la educación de los hijos, evidenciado en el mejor rendimiento académico.

5. Recomendamos se elabore con celeridad propuestas de Gestión Pedagógica para integrar las TIC a los padres y se estandarice aplicándola en las familias industrialinas, familias nacionales e internacionales.

6. En Perú, claramente se evidencia brechas digitales entre las zonas urbanas y rurales que constituye un gran reto a superar para lograr los objetivos de aprendizaje planteados. 
7. La dotación de Tablets, la subvención por parte del Estado de los servicios de Internet para la comunidad en conjunto ofrece posibilidades tangibles de desarrollo a través de la educación y un trabajo unificado de la comunidad educativa.

8. Las generaciones digitales requieren orientación pertinente para la adecuada integración de las TIC a su vida cotidiana, usando aquella información confiable que aporte a su aprendizaje.

\section{LISTA DE REFERENCIAS}

Colás P. Contreras J. Universidad de Sevilla. La participación de las familias en los centros escolares de Educación Primaria. P. 496. España 2013. Consulta 27.05.20 https://revistas.um.es/rie/article/view/171031/158201

Lozano J. Ballesta J. Alcaraz S. Cerezo M. Universidad de Sevilla. Las tecnologías de la información y la comunicación en la relación familia-escuela P. 14. España 2014. Consulta 02.06 .20 https://revistascientificas.us.es/index.php/fuentes/article/view/2574

Hernández A. López P. Sánchez S. Universidad de Alcalá. La comunicación en la familia a través de las TIC. Percepción de los adolescentes P. 52. España 2014. Consulta 01.06 .20 https://ebuah.uah.es/dspace/bitstream/handle/10017/21126/Comunicaci\%c3\%b3 n\%20Hern\%c3\%a1ndez.pdf?sequence $=1 \&$ is Allowed $=\mathrm{y}$

García A. Barreto M. Instituto de Estudios Peruanos. El uso, apropiación e impacto de las TIC por las mujeres rurales jóvenes en el Perú P. 265. Perú 2014. Consulta 28.05.20 http://revista-redes.hospedagemdesites.ws/index.php/revistaredes/article/view/305/344

Llevot N. Bernat O. Universidad de Lleida. La participación de las familias en la escuela: factores clave P. 67. España 2015. Consulta 30-05-2020. https://ojs.uv.es/index.php/RASE/article/viewFile/8761/8304

Macià, M. Universidad de Lleida. La comunicación familia-escuela: el uso de las TIC en los centros de primaria. P. 81, España 2016. Consulta 31-05-2020. https://revistas.um.es/reifop/article/view/245841

Garreta J. Universidad de Lleida. Fortalezas y debilidades de la participación de las familias en la escuela. Perspectiva educacional. P. 153, España 2016. Consulta 01-06-20. 
https://www.researchgate.net/publication/304627500_Garreta_J_2016_Fortaleza s_y_debilidades_de_la_participacion_de_las_familias_en_la_escuela_Perspectiv a_educacional_552_141-157/link/5775649f08ae1b18a7dfdf38/download

Garreta J. Universidad de Lleida. Fortalezas y debilidades de la participación de las familias en la escuela. Perspectiva educacional. P. 153, España 2016. Consulta 01-06-20.

https://www.researchgate.net/publication/304627500_Garreta_J_2016_Fortaleza s_y_debilidades_de_la_participacion_de_las_familias_en_la_escuela_Perspectiv a_educacional_552_141-157/link/5775649f08ae1b18a7dfdf38/download

Pedraza C. Amado O. Munévar P (2019). Universidad Nacional Abierta y a Distancia. Apropiación de dispositivos móviles en educación: una experiencia de sistematización sobre uso pedagógico de TIC en tabletas. P. 28 Colombia. Consulta 30-05-2020 en https://revistas.usantotomas.edu.co/index.php/riiep/article/view/5457

Ramírez A. Salcines I. Gonzáles N. (2020). Universidad de Córdoba. Los dispositivos móviles en el hogar. Interés formativo de las familias españolas. P. 54,55,56 España. Consulta 31-05-2020

en http://revistas.uned.es/index.php/reop/article/view/27286

Britez M. (2020). Universidad Nacional del Este Paraguay. La educación ante el avance del COVID-19 en Paraguay. Comparativo con países de la Triple Frontera. Paraguay. Consulta 31-05-2020 en https://preprints.scielo.org/index.php/scielo/preprint/view/22/579

Programme for International Student Assessment - PISA (2018), consultado el 04-06-20 en https://www.oecd.org/pisa/pisaenespaol.htm\#Unión Internacional de Telecomunicaciones (UIT), organismo especializado de las Naciones Unidas para las tecnologías de la información y la comunicación-TIC (2016), consultado el 30-05-20 en https://www.itu.int/es/mediacentre/Pages/2016-PR30.aspx

MINEDU Ministerio de Educación del Perú, portal institucional https://www.gob.pe/minedu, visitado em Octubre del 2020. 